\title{
Objective Measurement of Impact of Bench Laparoscopic Training in Novices
}

\author{
Prashanth Annayyanapalya Thimmegowda ${ }^{1}$, Krish Lakshman ${ }^{2}$, Rajashekara Reddy ${ }^{1}$ Sachin Nale ${ }^{1}$, \\ Ravishankar ${ }^{1}$ \\ ${ }^{1}$ Department of General Surgery, Sagar Hospital, Jayanagar, Bangalore, Karnataka, India \\ ${ }^{2}$ Department of General Surgery, Shanthi Hospital and Research Centre (SHRC), Jayanagar, Bangalore, \\ Karnataka, India
}

Correspondence to: Dr. Prashanth Annayyanapalya Thimmegowda; email: at.prashanth@gmail.com

Received: 19 Sep 2020; Revised:13 Apr 2021; Accepted: 15 Apr 2021; Available online: 23 Apr 2021

\begin{abstract}
Background: We are currently in the era of laparoscopic surgery. It has gained popularity in the last few decades because of its well-known advantages. Laparoscopy requires different skills from those of open surgery. In a paradigm shift, learning basic surgical skills is now performed more in the skills laboratory than in the operation theater. However, there is a lack of reliable training and assessment tools for laparoscopic surgical skills. This study aimed to objectively assess the effect of bench laparoscopic training in novices. Methods: This prospective study was conducted at the Clinical Skills Centre of Bangalore Medical College and Research Institute (BMCRI) in Bangalore, India. Sixty interns with no previous experience in laparoscopy were included. They underwent supervised training on the box trainer for 3 days, 2 hours a day, in basic surgical tasks, including pointing dots, joining straight lines, joining curved lines, picking objects, peg transfer, and
\end{abstract}

\section{Introduction}

Laparoscopic surgery has gained popularity in the last few decades and has been steadily replacing open standard techniques in several procedures because of its advantages such as minimal surgical trauma, early postoperative recovery, shorter hospital stays, and better cosmesis (1-3). Learning laparoscopic surgical skills require a different set of skills due to diminished tactile feedback, need for hand-to-eye coordination, the fulcrum effect caused by trocars and long instruments, circle cut. All participants were assessed objectively in a virtual reality (VR) simulator before and after training. The objective outcomes measured were time taken, distance traveled, and error scores given by the VR simulator metrics. Results: The novices showed statistically significant improvement in all the tasks after the training compared with their skill levels before the training. Conclusion: Structured short-term training significantly improves basic laparoscopic surgery skills.

Keywords: Bench laparoscopic training; Box trainer; Virtual reality simulator; Novices training; Endo training

Ann Afr Surg. 2021; 18(3): 170-175

DOI: http://dx.doi.org/10.4314/aas.v18i3.8

Conflicts of Interest: None

Funding: None

(C) 2021 Author. This work is licensed under the Creative Commons Attribution 4.0 International License.

and accommodation for lack of depth perception with $2 \mathrm{D}$ vision (4).

Acquiring basic surgical skills outside the operation theater (OT) is ideal (5) because valuable OT time is not wasted and concerns for patient safety are avoided. The old dictum "see one, do one, teach one" does not apply anymore. Simulation modules play a very important role in mastering these skills, thereby flattening the learning curve. Different simulation models are currently available, such as box trainer, virtual reality (VR) 
simulator, augmented reality (AR) simulator, animal models, and cadaver models. Among them, only VR and AR simulators provide objective metrics and real-time feedback to trainees (6).

Box trainers are relatively cheap and have realistic haptic feedback, which VR simulators lack. Compared with VR simulators, box trainers have the advantage of using standard laparoscopic instruments. The biggest advantage of a VR simulator is that it provides objective metrics, which helps train students better. However, in evaluating laparoscopic surgical skills, both the box trainer and VR simulator correlate positively with each other (7). In this prospective trial, we investigated the impact of bench laparoscopic training on basic laparoscopic surgical skills using the box trainer, and objective assessment of the said training was carried out in a VR simulator.

\section{Methods}

This prospective study was conducted at a clinical skills center in Bangalore, India, from January to March 2018. The study protocol was approved by the hospital ethics committee. Informed consent was obtained from all individual participants included in the study.

We included 60 interns from Bangalore Medical College and Research Institute (BMCRI) with no experience in laparoscopic surgeries. The novices were assessed on a VR simulator before they started their training on the box trainer. The VR trainer is an indigenously designed and built trainer (BIGSOLV Laparo simulator; Sri Dutt Technologies, Bangalore, India) that has been validated to measure basic laparoscopic skills (8).

The interns underwent supervised training on the box trainer for 3 days, 2hours each day. The training included standardized basic surgical tasks, including pointing dots, joining straight lines, joining curved lines, picking objects, peg transfer, and circle cut. The tasks were of progressive complexity. The participants standardized skills were assessed in a VR simulator immediately after the training. The primary outcomes of interest were time taken to complete the tasks, total path length, and error scores. These data were objectively given by the VR trainer used in the study.

\section{Pre-training assessment in the VR simulator}

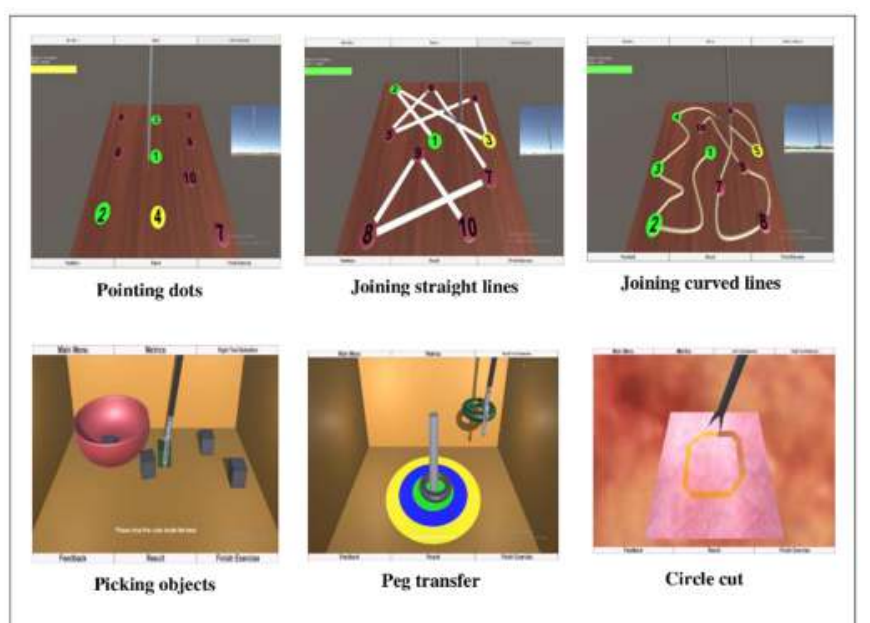

Figure 1. shows the images of the various tasks as seen in the VR simulator.

In task 1, pointing dots, trainees had to move the tool sequentially from 1 to 10 . In task 2 , joining straight lines, the tool had to be moved sequentially from 1 to 10 along the straight path provided. In task 3 , joining curved lines, the tool had to be moved along the curved path provided. For tasks 1, 2, and 3, a needle tool was used, and these tasks were mainly aimed at improving the hand-to-eye coordination. The points have to be traversed in an incremental manner starting from 1 and ending at 10 . Any effort to follow a non-incremental approach was counted as an error.

Task 4, picking objects, the trainee was supposed to pick up the cube using the tool and transfer it into the bowl. While transferring the cube, care was to be taken to see that the cube was not dropped outside the bowl. Picking up the cube with the tool and releasing it elsewhere apart from the bowl was counted as an error.

Task 5, peg transfer, consisted of a torus and a peg, and the trainee was asked to pick up the torus using the tool and transfer it over the peg. While transferring the peg, care was to be taken to see that the torus will not touch the peg, and doing so resulted in an error. Picking up the torus with the tool and releasing it elsewhere apart from the peg was counted as an error.

For tasks 4 and 5, a grasper tool was used, and these tasks were aimed at giving the user a higher degree of hand-to-eye coordination than that in tasks $1-3$, with respect to handling objects in a $3 \mathrm{D}$ space. In task 6 , circle 
cut, a section of the tissue highlighted in yellow had to be cut using a scissor. It involved cutting the entire highlighted region to end the exercise. Cutting outside the highlighted region was counted as an error.

The objective metrics for the time taken to complete the tasks, total path length, and error scores for all the performed tasks as given by the VR simulator were recorded. Fig. 2 shows the images of the various tasks as seen on the box trainer.

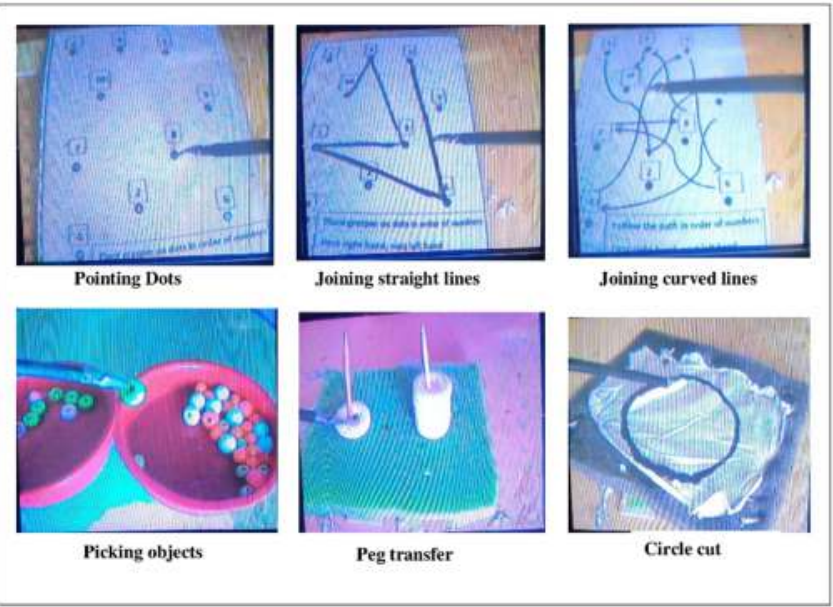

Figure 2. Images of the various tasks as seen on the box trainer.

\section{Training on the box trainer}

After the pre-training assessment, all the trainees underwent supervised training by consultant laparoscopic surgeons with a minimum experience of 5 years. The same tasks that were included in the VR simulator assessment were included in the box training

\section{Post-training assessment in the VR simulator}

The trainees' post-box training tasks, which were the same as those in the pre-training, were assessed in the VR simulator. The objective metrics given by the VR simulator were again recorded.

\section{Statistical analysis}

Data were analyzed using Student's paired t-test. A pvalue $<0.05$ was considered statistically significant. Data was analyzed using SPSS for Windows version 20.0 (IBM Corp., Armonk, NY, USA) (Table 1).

\section{Results}

The mean time taken to complete the tasks was significantly shorter in post-training than that in pretraining in all the tasks ( $\mathrm{p}<0.05$ for all the tasks; Fig. $3)$. The mean distance traveled in all the tasks in posttraining were significantly shorter than that in pretraining $(p<0.05$; Fig. 4).Regarding the error score outcome, the trainees made significantly fewer errors in post-training that in pre-training, except in the picking objects and circle cut tasks(Fig. 5). In the picking objects task, the mean error scoreswere 3 in pre-training and 4 in post-training, but the difference was not statistically significant $(\mathrm{p}=0.35)$. In the circle cut task, the mean error score was 3 in pre-training and 10 in post-training, and the difference was statistically significant $(p=0.01)$.

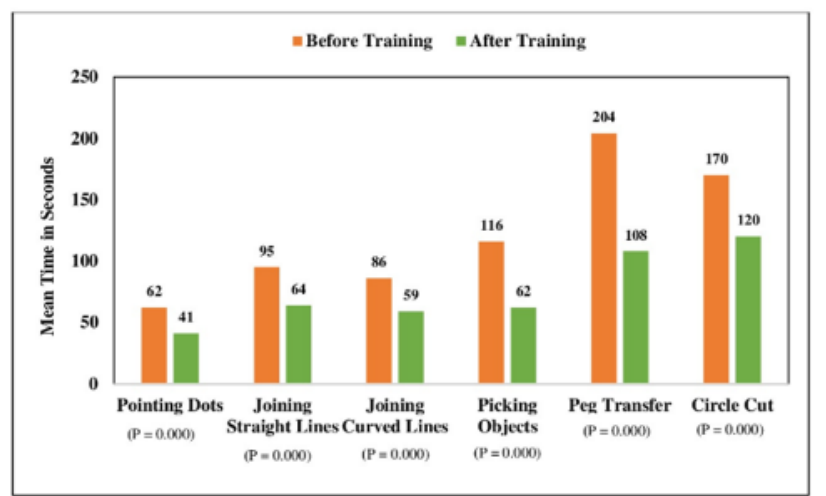

Figure 3. Mean time taken to complete the tasks

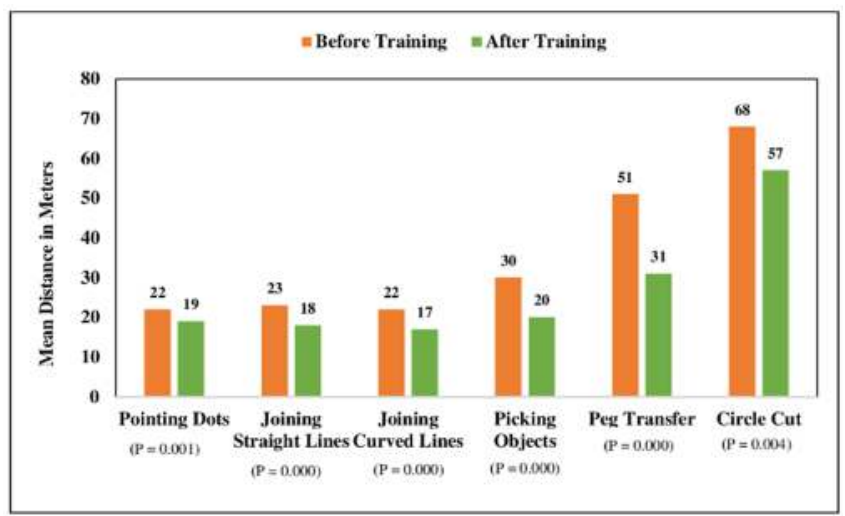

Figure 4. Mean distance traveled in all the tasks

\section{Discussion}

We are currently in the era of laparoscopic surgery. It had gained popularity in the last few decades because of its well-known advantages. 


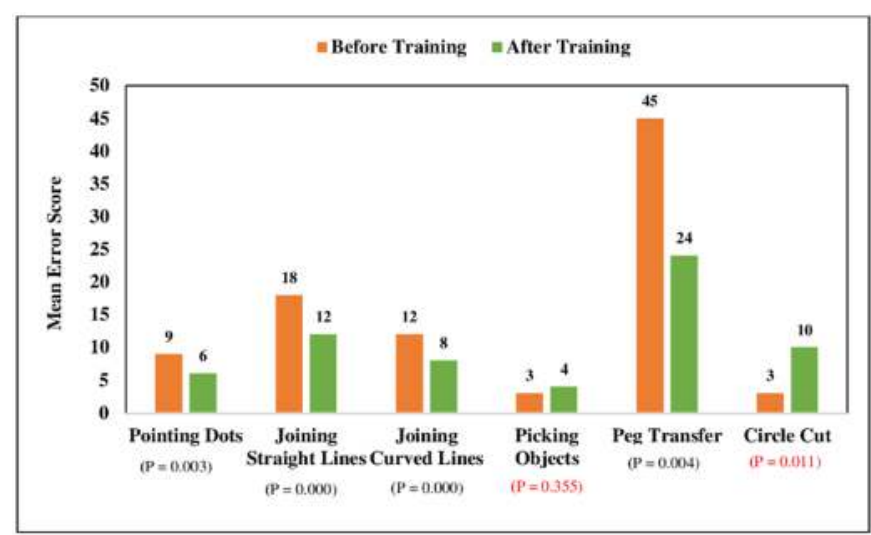

Figure 5. Mean error score outcome

Although its scope and application are expanding at a rapid rate, we lack reliable training and assessment tools (9). Training for laparoscopic surgical skills is different from that of open surgery because laparoscopic surgery needs good hand-to-eye coordination, good manual dexterity in using long instruments, and good accommodation for lack-of-depth perception with 2D vision (10). The acquisition of these skills in the operating room is time-consuming and is also a safety concern for the patient.

Box trainers are inexpensive and are easy to maintain. Their use has been shown to improve hand-to-eye coordination, ambidexterity, manipulation of long instruments in a limited space, depth perception, and familiarization with reduced haptic feedback using simple basic exercises $(11,12,13)$. Retention of these skills, especially for basic laparoscopic skills (14-18), has also been established.

However, box trainers cannot automatically provide metrics for objective evaluation of the tasks performed. We need to have an assessor that objectively evaluates the skills of a trainee. This assessment is subjective and can lead to inter- and intra-observer variations. In our study, we eliminated this bias by using a VR simulator for the assessment.

Many studies have shown that the efficacy of acquiring basic laparoscopic skills in VR simulator and box trainer are comparable and the skills acquired in box trainer can be reproducible in a VR simulator and vice versa $(19,20)$. Similar studies done to assess the effectiveness of box trainer in acquiring laparoscopic skills have trained students for a period ranging from 2 hours to 6 days in some studies. Basic laparoscopic skills such as depth perception, hand-to-eye coordination, handling of laparoscopic instruments, and bimanual manipulation were tested (21-23). The training capabilities of VR trainer and box trainer are comparable, and there is no difference in the skill acquisition between these two trainers (24). A good correlation exists between both these trainers in assessing laparoscopic skills (25). Our study showed that those who underwent training for basic laparoscopic skills on the box trainer performed better after the training, as measured objectively on a VR trainer. The improvement was obvious and comprehensive as far as the time taken to perform and the distance traveled to complete the tasks were concerned. Regarding the error score, the picking objects and circle cut tasks showed conflicting results. Although the former was not statistically significant, the latter showed that the error score was lower in the pretraining phase, which is a surprising result. We can only speculate the causes for the same, such as the novices were not concentrating enough on these tasks during the training period or had a casual attitude while doing the exercise on the VR trainer during assessment, or, less likely, the box trainer has a limitation in providing skill acquisition in more complex tasks.

The limitations in our study include the following. Although the training was supervised, there was no way we could enforce uniformity in all the subjects as far as carrying out the tasks was concerned. Furthermore, although the overall time spent on the training was uniform, it was difficult to enforce training time for individual exercises. Hence, the next step would be to establish whether this basic skill acquired in the skills laboratory can be retained over a period of time (weeks to months) and translated into improved performance at an actual operation in the OT.

\section{Conclusion}

Structured short-term training on box trainers significantly improves the basic skills in laparoscopic surgery. Structured training on a box trainer in the skills laboratory should been integral part of post-graduate training. 


\section{References}

1. Bruhat MA, Pouly JL. Endoscopic treatment of ectopic pregnancies. Curr OpinObstetGynecol. 1993;5(2):260266.

2. Keus F, Broeders IA, Van Laarhoven CJ. Surgical aspects of symptomatic cholecystolithiasis and acute cholecystitis. Best PractResClinGastroenterol. 2006;20(6):1031-1051.

3. Rosenmüller $\mathrm{M}$, Haapamäki $\mathrm{MM}$, Nordin $\mathrm{P}$, et al. Cholecystectomy in Sweden 2000-2003: a nationwide study on procedures, patient characteristics, and mortality. BMC Gastroenterol. 2007;7(1):35.

4. Gallagher AG, McClure N, McGuigan J, et al. Virtual reality training in laparoscopic surgery: a preliminary assessment of minimally invasive surgical trainer virtual reality (MIST-VR). Endoscopy. 1999;31:310 -313.

5. Papanikolaou IG, Haidopoulos D, Paschopoulos M, et al. Changing the way we train surgeons in the 21 st century: a narrative comparative review focused on box trainers and virtual reality simulators. EurJObstetGynecolReprodBiol. 2019;235:13-18.

6. Nagendran M, Gurusamy KS, Aggarwal R, et al. Virtual reality training for surgical trainees in laparoscopic surgery. Cochrane Database SystRev. 2013(8):CD006575.

7. Newmark J, Dandolu V, Milner R, et al. Correlating virtual reality and box trainer tasks in the assessment of laparoscopic surgical skills. Am J Obstet Gynecol. 2007;197(5):546.e1-e4.

8. Aspari AR, Ramesh V, Lakshman K. An indigenous virtual reality-based simulator - a tool in surgical training. Indian JSurg. 2020:1-7.

9. Emken JL, McDougall EM, Clayman RV. Training and assessment of laparoscopic skills. JSLS. 2004;8(2):195.

10. Ahlberg G, Enochsson L, Gallagher AG, et al. Proficiencybased virtual reality training significantly reduces the error rate for residents during their first 10 laparoscopic cholecystectomies. Am J Surg. 2007;193(6):797-804.

11. Munz Y, Kumar BD, Moorthy K, et al. Laparoscopic virtual reality and box trainers: is one superior to the other? Surg Endosc. 2004;18(3):485-494.

12. Caban AM, Guido C, Silver M, et al. Use of collapsible box trainer as a module for resident education. JSLS. 2013;17(3):440-444.

13. Schreuder HW, Van Den Berg CB, Hazebroek EJ, et al. Laparoscopic skills training using inexpensive box trainers: which exercises to choose when constructing a validated training course. BJOG. 2011;118(13):15761584.

14. Supe A, Prabhu R, Harris I, et al. Structured training on box trainers for first year surgical residents: does it improve retention of laparoscopic skills? A randomized controlled study. J Surg Educ. 2012;69(5):624-632.
15. Reznick RK, MacRae H. Teaching surgical skillschanges in the wind. NEnglJMed. 2006;355(25):26642669.

16. Brinkman WM, Havermans SY, Buzink SN, et al. Single versus multimodality training basic laparoscopic skills. Surg Endosc. 2012;26(8):2172-2178.

17. Chummun K, Burke JP, O’Sullivan R, et al. The influence of a 'take home' box trainer on laparoscopic performance for gynaecological surgeons. Gynecol Surv. 2012;9(3):303-308.

18. van Empel PJ, Verdam MG, Strypet M, et al. Voluntary autonomous simulator based training in minimally invasive surgery, residents' compliance and reflection. J Surg Educ. 2012;69(4):564-570.

19. Diesen DL, Erhunmwunsee L, Bennett KM, et al. Effectiveness of laparoscopic computer simulator versus usage of box trainer for endoscopic surgery training of novices. JSurg Educ. 2011;68(4):282-289.

20. Mulla M, Sharma D, Moghul M, et al. Learning basic laparoscopic skills: a randomized controlled study comparing box trainer, virtual reality simulator, and mental training. J Surg Educ. 2012;69(2):190-195.

21. Vitish-Sharma P, Knowles J, Patel B. Acquisition of fundamental laparoscopic skills: is a box really as good as a virtual reality trainer?Int J Surg. 2011;9(8):659-661.

22. Mohammadi Y, Lerner MA, Sethi AS, et al. Comparison of laparoscopy training using the box trainer versus the virtual trainer. JSLS. 2010;14(2):205.

23. Dhariwal AK, Prabhu RY, Dalvi AN, et al. Effectiveness of box trainers in laparoscopic training. J Minim Access Surg. 2007;3(2):57.

24. Katz R. Methods of training using pelvic trainers. Curr Urol Rep. 2006;7(2):100-106.

25. Madan AK, Frantzides CT. Substituting virtual reality trainers for inanimate box trainers does not decrease laparoscopic skills acquisition. JSLS. 2007;11(1):87-89. 